\title{
Discovery of new depigmenting compounds and their efficacy to treat hyperpigmentation: evidence from in vitro study
}

\begin{abstract}
Human skin pigmentation is a result of constitutive and facultative pigmentation. Facultative pigmentation is frequently stimulated by UV radiation, pharmacologic drugs, and hormones whereby leads to the development of abnormal skin hyperpigmentation. To date, many stateof-art depigmenting compounds have been studied using in vitro model to treat hyperpigmentation problems for cosmetic dermatological applications; little attention has been made to compare the effectiveness of these depigmenting compounds and their mode of actions. In this present article, new and recent depigmenting compounds, their melanogenic pathway targets, and modes of action are reviewed. This article compares the effectivenes s of these new depigmenting compounds to modulate several melanogenesis-regulatory enzymes and proteins such as tyrosinase (TYR), TYR-related protein-1 (TRP1), TYR-related protein-2 (TRP2), microphthalmia-associated transcription factor (MITF), extracellular signal-regulated kinase (ERK) and N-terminal kinases (JNK) and mitogen-activated protein kinase p38 (p38 MAPK). Other evidences from in vitro assays such as inhibition on melanosomal transfer, proteasomes, nitric oxide, and inflammation-induced melanogenesis are also highlighted. This article also reviews analytical techniques in different assays performed using in vitro model as well as their advantages and limitations. This article also provides an insight on recent finding and re-examination of some protocols as well as their effectiveness and reliability in the evaluation of depigmenting compounds. Evidence and support from related patents are also incorporated in this present article to give an overview on current patented technology, latest trends, and intellectual values of some depigmenting compounds and protocols, which are rarely highlighted in the literatures.
\end{abstract}

Keyword: Coculture; In vitro; Keratinocyte; Melanin; Melanocyte; Melanoma; Pigmentation; Tyrosinase 